\title{
Unbounded solutions of an iterative-difference equation
}

\section{Lin Li}

College of Mathematics, Physics and Information Engineering Jiaxing University, P. R. China email: mathll@163.com
Pingping Zhang

Department of Mathematics, Binzhou University, P. R. China email: zhangpingpingmath@163.com

Abstract. Unbounded solutions for the iterative-difference equation

$$
f^{2}(x)=\lambda f(x+a)+\mu x, x \in \mathbb{R},
$$

have been considered in [Continuous solutions of an iterative-difference equation and Brillouët problem, Publ. Math. Debrecen, 78 (2011), 613$624]$, where $\lambda, \mu, a$ are real constants. In this paper, we continue to study the solutions not being included there, and further give the convex and concave ones. Finally, continuous solutions of this equation with an extra item were also given, which continuously depend on the parameter $a$.

\section{Introduction}

The iterative-difference equation

$$
f^{2}(x)=f(x+a)-x, \quad x \in \mathbb{R}
$$

proposed by N. Brillouët-Belluot [2], was deduced from the equation $x+f(y+$ $f(x))=y+f(x+f(y))$, a special form of the functional equation

$$
x+g(y+f(x))=y+f(x+g(y))
$$

2010 Mathematics Subject Classification: 39B12, 37E05

Key words and phrases: iterative-difference equation; existence; unbounded solution; convex solution; concave solution 
investigated in $[8,12]$. Although the nonexistence of continuous solutions and existence of special solutions were discussed $[1,7,11]$, it is still treated as a difficult problem to find continuous solutions of Eq.(1). In 2010, as a generalized form of (1), the equation

$$
f^{2}(x)=\lambda f(x+a)+\mu x
$$

where $\lambda, \mu, a$ are real and $a \lambda \neq 0$ was considered on a compact interval by N. Brillouët-Belluot and W. Zhang ([3]). In fact, when $a=0$ all continuous solutions were presented in 1974 by S. Nabeya ([10]) and continuous solutions defined on a real Hausdorff topological linear space were studied by J. Dhombres ([5]) in the case that $\lambda+\mu=1$. The authors in [3] not only searched all affine solutions, but also constructed piecewise continuous solutions of Eq.(2). Later, Y. Zeng and W. Zhang [17] investigated the solutions on the whole $\mathbb{R}$ and gave the unbounded continuous solutions of Eq.(2) in some cases, where the following cases are still open: (E1) $|\lambda| \in(0,1]$; (E2) $|\lambda| \in(1,2]$ and $|\mu| \in[|\lambda|-1,+\infty) ;($ E3 $)|\lambda| \in(2,+\infty)$ and $|\mu| \in\left(\lambda^{2} / 4,+\infty\right)$. Although when $\lambda=1$ and $\mu \leq-1$, a special case of (E1), was solved (see Theorem 1 in [17]), the existence or nonexistence of solutions almost remains unknown for the cases (E1)-(E3). This paper is a continuation of studying Eq.(2) on the whole $\mathbb{R}$. We first consider the continuous solutions with the form of

$$
f(x)=\alpha x+f_{1}(x)
$$

where $\alpha$ is a real constant and $f_{1}: \mathbb{R} \rightarrow \mathbb{R}$ is continuous. By using the Banach fixed point principle, the existence and uniqueness of solutions including convex and concave ones, are given for cases (E2)-(E3). Finally, in order to show the characterization of solutions for case (E1), we investigate the continuous solutions of Eq.(2) with an extra item and prove that those solutions continuously depend on the parameter a.

\section{Existence of unbounded solutions}

By replacing the function $f(x)$ with $g(x):=\frac{1}{a} f(a x)$, it suffices to consider the solutions of Eq.(2) with $a=1$, i.e.,

$$
f^{2}(x)=\lambda f(x+1)+\mu x, \quad x \in \mathbb{R} .
$$

Further, substituting (3) into (4) we obtain

$$
\begin{aligned}
f^{2}(x-1) & =\alpha\left(\alpha(x-1)+f_{1}(x-1)\right)+f_{1}\left(\alpha(x-1)+f_{1}(x-1)\right) \\
& =\lambda \alpha x+\lambda f_{1}(x)+\mu(x-1),
\end{aligned}
$$


and then

$$
f_{1}(x)=\frac{\alpha^{2}-\lambda \alpha-\mu}{\lambda} x+\frac{\alpha}{\lambda} f_{1}(x-1)+\frac{1}{\lambda} f_{1}\left(f_{1}(x-1)+\alpha x-\alpha\right)-\frac{\alpha^{2}-\mu}{\lambda} .
$$

Let

$$
\Phi(\mathbb{R} ; \mathrm{L})=\{f: \mathbb{R} \rightarrow \mathbb{R} \mid \operatorname{Lipf} \leq \mathrm{L}\}
$$

for a given real constant $L>0$. We note that $\Phi(\mathbb{R} ; \mathrm{L})$ is a complete metric space equipped with the metric $d(f, g):=\sup _{x \in \mathbb{R}}|f(x)-g(x)|$.

Theorem 1 For a given $\alpha \in \mathbb{R}$, Eq. (4) has a unique solution $\mathrm{f}(\mathrm{x})=\alpha \mathrm{x}+$ $\mathrm{f}_{1}(\mathrm{x})$, where $\mathrm{f}_{1} \in \Phi(\mathbb{R} ; \mathrm{L})$ provided that $\mathrm{L}>0$ and

$$
\begin{array}{r}
\left|\frac{\alpha^{2}-\lambda \alpha-\mu}{\lambda}\right|+2\left|\frac{\alpha}{\lambda}\right| \mathrm{L}+\frac{1}{|\lambda|} \mathrm{L}^{2} \leq \mathrm{L}, \\
\frac{|\alpha|+1+\mathrm{L}}{|\lambda|}<1 .
\end{array}
$$

Proof. For a given $\mathrm{L}>0$, define a mapping $\mathcal{F}: \Phi(\mathbb{R} ; \mathrm{L}) \rightarrow \mathrm{C}^{0}(\mathbb{R})$ by

$$
\mathcal{F} f_{1}(x)=\frac{\alpha^{2}-\lambda \alpha-\mu}{\lambda} \chi+\frac{\alpha}{\lambda} f_{1}(x-1)+\frac{1}{\lambda} f_{1}\left(f_{1}(x-1)+\alpha x-\alpha\right)-\frac{\alpha^{2}-\mu}{\lambda},
$$

where $f_{1} \in \Phi(\mathbb{R} ; L)$. It follows from (5) that for every $x, y \in \mathbb{R}$,

$$
\begin{aligned}
\left|\mathcal{F} f_{1}(x)-\mathcal{F} f_{1}(y)\right|= & \mid \frac{\alpha^{2}-\lambda \alpha-\mu}{\lambda} x+\frac{\alpha}{\lambda} f_{1}(x-1)+\frac{1}{\lambda} f_{1}\left(f_{1}(x-1)+\alpha x-\alpha\right) \\
& -\frac{\alpha^{2}-\lambda \alpha-\mu}{\lambda} y+\frac{\alpha}{\lambda} f_{1}(y-1)+\frac{1}{\lambda} f_{1}\left(f_{1}(y-1)+\alpha y-\alpha\right) \mid \\
\leq & \left|\frac{\alpha^{2}-\lambda \alpha-\mu}{\lambda}\right||x-y|+\left|\frac{\alpha}{\lambda}\right| L|x-y| \\
& +\frac{1}{|\lambda|} L\left|\alpha x+f_{1}(x-1)-\alpha y-f_{1}(y-1)\right| \\
\leq & \left(\left|\frac{\alpha^{2}-\lambda \alpha-\mu}{\lambda}\right|+2\left|\frac{\alpha}{\lambda}\right| L+\frac{1}{|\lambda|} L^{2}\right)|x-y| \leq L|x-y| .
\end{aligned}
$$


This implies that $\mathcal{F}$ maps $\Phi(\mathbb{R} ; \mathrm{L})$ into itself. Furthermore, for every $f_{1}, f_{2} \in$ $\Phi(\mathbb{R} ; \mathrm{L})$ we have

$$
\begin{aligned}
& \left|\mathcal{F} f_{1}(x)-\mathcal{F} f_{2}(x)\right| \\
& =\left|\frac{\alpha}{\lambda} f_{1}(x-1)+\frac{1}{\lambda} f_{1}\left(f_{1}(x-1)+\alpha x-\alpha\right)-\frac{\alpha}{\lambda} f_{2}(x-1)-\frac{1}{\lambda} f_{2}\left(f_{2}(x-1)+\alpha x-\alpha\right)\right| \\
& \leq\left|\frac{\alpha}{\lambda}\right| d\left(f_{1}, f_{2}\right)+\frac{1}{|\lambda|}\left|f_{1}\left(f_{1}(x-1)+\alpha x-\alpha\right)-f_{2}\left(f_{2}(x-1)+\alpha x-\alpha\right)\right| \\
& \leq\left|\frac{\alpha}{\lambda}\right| d\left(f_{1}, f_{2}\right)+\frac{1}{|\lambda|}\left|f_{1}\left(f_{1}(x-1)+\alpha x-\alpha\right)-f_{2}\left(f_{1}(x-1)+\alpha x-\alpha\right)\right| \\
& +\frac{1}{|\lambda|}\left|f_{2}\left(f_{1}(x-1)+\alpha x-\alpha\right)-f_{2}\left(f_{2}(x-1)+\alpha x-\alpha\right)\right| \\
& \leq \frac{(|\alpha|+1+L)}{|\lambda|} d\left(f_{1}, f_{2}\right), \forall x \in \mathbb{R}
\end{aligned}
$$

i.e.,

$$
\mathrm{d}\left(\mathcal{F} \mathrm{f}_{1}, \mathcal{F} \mathrm{f}_{2}\right) \leq \frac{(|\alpha|+1+\mathrm{L})}{|\lambda|} \mathrm{d}\left(\mathrm{f}_{1}, \mathrm{f}_{2}\right)
$$

Hence, $\mathcal{F}: \Phi(\mathbb{R} ; \mathrm{L}) \rightarrow \Phi(\mathbb{R} ; \mathrm{L})$ is a contraction if condition (6) holds. Therefore, by Banach's fixed point theorem, $\mathcal{F}$ has a unique fixed point in the class $\Phi(\mathbb{R} ; \mathrm{L})$.

Remark 1 In view of Theorem 1, the assumptions on $\alpha, \mathrm{L}, \lambda$ and $\mu$ are given by (5)-(6). Compared with Theorem 2 in [17], although our conditions seem complicated because of the inequalities between $\alpha, \lambda, \mu$, cases $(\mathbf{E 2 )}-(\mathbf{E 3 )}$ in Theorem 1 were not considered in [17].

Example 1 Take $\alpha=0.4, \lambda=-2, \mu=1$ and $\mathrm{L}=0.28$.

It is easy to see that $\lambda, \mu$ satisfy (E2). Moreover,

$$
\left|\frac{\alpha^{2}-\lambda \alpha-\mu}{\lambda}\right|+2\left|\frac{\alpha}{\lambda}\right| \mathrm{L}+\frac{1}{|\lambda|} \mathrm{L}^{2}=0.1712<0.28, \frac{|\alpha|+1+\mathrm{L}}{|\lambda|}=0.84<1,
$$

which implies that (5)-(6) hold. Therefore, Eq. (4) has a unique solution $f(x)=$ $\frac{2}{5} x+f_{1}(x)$, where $f_{1} \in \Phi\left(\mathbb{R} ; \frac{7}{25}\right)$.

Example 2 Choose $\alpha=0.5, \lambda=-2.2, \mu=1.25$, and $\mathrm{L}=0.3$. 
One can check that $\lambda, \mu$ fulfills case (E3). Furthermore,

$$
\left|\frac{\alpha^{2}-\lambda \alpha-\mu}{\lambda}\right|+2\left|\frac{\alpha}{\lambda}\right| \mathrm{L}+\frac{1}{|\lambda|} \mathrm{L}^{2}<0.224<\mathrm{L}, \quad \frac{|\alpha|+1+\mathrm{L}}{|\lambda|}<0.82<1 .
$$

Thus, conditions (5)-(6) in Theorem 1 are satisfied and Eq. (4) has a unique solution $f(x)=\frac{1}{2} x+f_{1}(x)$ for $f_{1} \in \Phi\left(\mathbb{R} ; \frac{3}{10}\right)$.

In what follows, we turn to consider the convex and concave solutions of Eq. (4). A function $f$ is convex (concave) if $f(t x+(1-t) y) \leq t f(x)+(1-t) f(y)$ for all $x, y \in \mathbb{R}$ and $t \in[0,1]$ (if the reverse of the inequality holds). Let

$$
\begin{aligned}
\Phi^{c v}(L) & =\{f \in \Phi(\mathbb{R} ; L): f \text { is convex and increasing }\} . \\
\Phi^{c c}(L) & =\{f \in \Phi(\mathbb{R} ; L): f \text { is concave and increasing }\} .
\end{aligned}
$$

Clearly, $\Phi^{\mathrm{cv}}(\mathrm{L})$ and $\Phi^{\mathrm{cc}}(\mathrm{L})$ are closed subsets of $\Phi(\mathbb{R} ; \mathrm{L})$, which are also complete metric spaces.

Theorem 2 Replace $\Phi(\mathbb{R} ; \mathrm{L})$ in Theorem 1 by $\Phi^{\mathrm{cv}}(\mathrm{L})\left(\Phi^{\mathrm{cc}}(\mathrm{L})\right)$ and suppose that the conditions of Theorem 1 hold. If $\lambda>0, \alpha>0$ and $\alpha^{2}-\lambda \alpha-\mu>0$, then Eq. (4) has a unique convex (concave) solution $f(x)=\alpha x+f_{1}(x)$, where $\mathrm{f}_{1} \in \Phi^{\mathrm{cv}}(\mathrm{L})\left(\Phi^{\mathrm{cc}}(\mathrm{L})\right)$.

Proof. It suffices to prove the case of convex, the proof for concave is similar. Firstly, we give some useful facts by the convexity of $f_{1}$. Note that for every $x, y \in \mathbb{R}$ and $t \in[0,1]$,

$$
\begin{aligned}
f_{1}(t x+(1-t) y-1) & =f_{1}(t(x-1)+(1-t)(y-1)) \\
& \leq t f_{1}(x-1)+(1-t) f_{1}(y-1),
\end{aligned}
$$

it follows that

$$
\begin{aligned}
& t\left(f_{1}(x-1)+\alpha x-\alpha\right)+(1-t)\left(f_{1}(y-1)+\alpha y-\alpha\right) \\
& =t \alpha x+\alpha(1-t) y-\alpha+t f_{1}(x-1)+(1-t) f_{1}(y-1) \\
& \geq t \alpha x+\alpha(1-t) y-\alpha+f_{1}(t(x-1)+(1-t)(y-1)) \\
& =t \alpha x+\alpha(1-t) y-\alpha+f_{1}(t x+(1-t) y-1) .
\end{aligned}
$$


Thus,

$$
\begin{aligned}
& f_{1}\left(t \alpha x+\alpha(1-t) y-\alpha+f_{1}(t x+(1-t) y-1)\right. \\
& \leq f_{1}\left(t\left(f_{1}(x-1)+\alpha x-\alpha\right)+(1-t)\left(f_{1}(y-1)+\alpha y-\alpha\right)\right) \\
& \leq t f_{1}\left(f_{1}(x-1)+\alpha x-\alpha\right)+(1-t) f_{1}\left(f_{1}(y-1)+\alpha y-\alpha\right)
\end{aligned}
$$

since $\boldsymbol{f}_{1}$ is increasing. From (8)-(9), for the function $\mathcal{F} \boldsymbol{f}_{1}$ defined in (7) we have

$$
\begin{aligned}
& \quad \mathcal{F} f_{1}(t x+(1-t) y) \\
& =\frac{\alpha^{2}-\lambda \alpha-\mu}{\lambda}(t x+(1-t) y)+\frac{\alpha}{\lambda} f_{1}(t x+(1-t) y-1) \\
& \quad+\frac{1}{\lambda} f_{1}\left(\alpha(t x+(1-t) y)-\alpha+f_{1}(t x+(1-t) y-1)-1\right)-\frac{\alpha^{2}+\alpha-\mu}{\lambda} \\
& \leq t \mathcal{F} f_{1}(x)+(1-t) \mathcal{F} f_{1}(y),
\end{aligned}
$$

which implies that $\mathcal{F} f_{1}$ is convex and increasing if $\lambda>0, \alpha>0, \alpha^{2}-\lambda \alpha-\mu>0$. Therefore, $\mathcal{F}$ maps $\Phi^{c v}(\mathrm{~L})\left(\Phi^{c c}(\mathrm{~L})\right)$ into itself, and the rest proof is same as that of Theorem 1.

Example 3 Take $\alpha=0.8, \lambda=2, \mu=-0.96$ and $\mathrm{L}=0.09$.

It is easy to see that

$$
\left|\frac{\alpha^{2}-\lambda \alpha-\mu}{\lambda}\right|+2\left|\frac{\alpha}{\lambda}\right| \mathrm{L}+\frac{1}{|\lambda|} \mathrm{L}^{2}<0.077<0.09, \quad\left|\frac{\alpha}{\lambda}\right|+\frac{1}{|\lambda|}+\mathrm{L}=0.99<1,
$$

and then the conditions in Theorem 2 are satisfied. Therefore, Eq. (4) has a unique solution $f(x)=\frac{4}{5} x+f_{1}(x)$ where $f_{1} \in \Phi^{c v}(L)$.

\section{$3 \quad$ Unbounded solutions with $\varepsilon(a)$}

In this section we discuss the continuous solutions of Eq. (2) with an extra item, that is,

$$
f^{2}(x)=\lambda f(x+a)+\mu x+\varepsilon(a)
$$

for $\lambda \in(0,1]$, where $\varepsilon: \mathbb{R} \rightarrow \mathbb{R}$ is continuous with respect to the parameter a. Clearly, Eq. (2) becomes a second order iterative equation when $a=0$, 
which has been extensively studied $[4,6,9,13,14,15,16,18,19]$. In what follows, our aim is to find a suitable continuous function $\varepsilon$ in order to give a construction of solutions. Here, we consider Eq. (10) under the hypothesis

$(\mathcal{H}) \lambda \in(0,1], \lambda+\mu=1$ and $\varepsilon(a):=(4-3 \lambda) a$.

The following Theorems are our main results in this section.

Theorem 3 Under hypothesis $(\mathcal{H})$, for $\mathrm{a}<0$ and each $\mathrm{x}_{0} \in(-\infty,+\infty)$, Eq.(10) has a continuous solution in $\left(-\infty, x_{0}\right]$. Moreover, the solution depends on arbitrarily chosen orientation-preserving homeomorphism $\mathrm{f}_{1}:\left[\mathrm{x}_{1}, \mathrm{x}_{0}\right] \rightarrow$ $\left[x_{2}, x_{1}\right]$, where $x_{1}:=x_{0}+2 a$ and $x_{2}:=\lambda\left(x_{1}+a\right)+\mu x_{0}+\varepsilon(a)$.

Theorem 4 Under hypothesis $(\mathcal{H})$, for $\mathrm{a}>0$ and each $\mathrm{x}_{0} \in(-\infty,+\infty)$, Eq. (10) has a continuous solution in $\left[\mathrm{x}_{0},+\infty\right)$. Moreover, the solution depends on arbitrarily chosen orientation-preserving homeomorphism $\mathrm{f}_{1}:\left[\mathrm{x}_{0}, \mathrm{x}_{1}\right] \rightarrow$ $\left[x_{1}, x_{2}\right]$, where $x_{1}:=x_{0}+2 a$ and $x_{2}:=\lambda\left(x_{1}+a\right)+\mu x_{0}+\varepsilon(a)$.

We only give a proof to Theorem 3 . Theorem 4 can be proved similarly. Proof. Let $x_{1}:=x_{0}+2 a$. Substituting $x_{1}$ and $\varepsilon(a)$ defined in $(\mathcal{H})$ into $x_{2}:=$ $\lambda\left(x_{1}+a\right)+\mu x_{0}+\varepsilon(a)$, we obtain $x_{2}=x_{1}+2 a$. This gives the fact that $x_{2}<x_{1}<x_{0}$. Then we extend these points to a sequence $\left(x_{n}\right)_{n=2}^{\infty}$ by the recurrence formula

$$
x_{n}=\lambda\left(x_{n-1}+a\right)+\mu x_{n-2}+\varepsilon(a) .
$$

We assert that $\left(x_{n}\right)_{n=2}^{\infty}$ is a strictly decreasing sequence in $\left(-\infty, x_{0}\right]$ satisfying

$$
x_{n}=x_{n-1}+2 a .
$$

In fact, (12) is trivial for $n=2$. Suppose that this claim holds for all positive integers $n \leq k$, where $k \geq 2$ is a certain integer. Then

$x_{k+1}=\lambda\left(x_{k}+a\right)+\mu x_{k-1}+\varepsilon(a)=\lambda\left(x_{k}+a\right)+\mu\left(x_{k}-2 a\right)+(4-3 \lambda) a=x_{k}+2 a$

by the fact $\lambda+\mu=1$. Thus, (12) is proved by induction.

The monotonicity of $\left(x_{n}\right)_{n=2}^{\infty}$ implies that this sequence is divergent, i.e.,

$$
\left(-\infty, x_{0}\right]=\bigcup_{n=1}^{\infty} I_{n}
$$

where $I_{n}:=\left[x_{n}, x_{n-1}\right]$. 
Choose an orientation-preserving homeomorphism $f_{1}: I_{1} \rightarrow I_{2}$ arbitrarily such that

$$
f_{1}\left(x_{0}\right)=x_{1}, \quad f_{1}\left(x_{0}+a\right)=x_{1}+a, \quad f_{1}\left(x_{1}\right)=x_{2} .
$$

Then for all $n \geq 2$ we recursively define

$$
f_{n}(x):=\lambda f_{n-1}\left(f_{n-1}^{-1}(x)+a\right)+\mu f_{n-1}^{-1}(x)+\varepsilon(a), \quad \forall x \in I_{n} .
$$

We assert that for each positive integer $n \geq 2$ mapping $f_{n}: I_{n} \rightarrow I_{n+1}$ is an orientation-preserving homeomorphism fulfilling

$$
f_{n}\left(x_{n-1}\right)=x_{n}, \quad f_{n}\left(x_{n-1}+a\right)=x_{n}+a, \quad f_{n}\left(x_{n}\right)=x_{n+1} .
$$

It is trivial for $n=2$. Actually,

$$
\begin{aligned}
& f_{2}\left(x_{1}\right)=\lambda f_{1}\left(f_{1}^{-1}\left(x_{1}\right)+a\right)+\mu f_{1}^{-1}\left(x_{1}\right)+\varepsilon(a)=\lambda f_{1}\left(x_{0}+a\right)+\mu x_{0}+\varepsilon(a)=x_{2}, \\
& \begin{aligned}
f_{2}\left(x_{1}+a\right) & =\lambda f_{1}\left(f_{1}^{-1}\left(x_{1}+a\right)+a\right)+\mu f_{1}^{-1}\left(x_{1}+a\right)+\varepsilon(a) \\
& =\lambda f_{1}\left(x_{0}+2 a\right)+\mu\left(x_{0}+a\right)+\varepsilon(a) \\
& =\lambda x_{2}+\mu\left(x_{0}+a\right)+(4-3 \lambda) a \\
& =x_{2}+a
\end{aligned}
\end{aligned}
$$

and

$$
f_{2}\left(x_{2}\right)=\lambda f_{1}\left(f_{1}^{-1}\left(x_{2}\right)+a\right)+\mu f_{1}^{-1}\left(x_{2}\right)+\varepsilon(a)=\lambda f_{1}\left(x_{1}+a\right)+\mu x_{1}+\varepsilon(a)=x_{3} .
$$

Suppose that the assertion is true for all integers $n \leq k$, where $k \geq 2$ is a certain integer. It is easy to see that $f_{k}$ is an orientation-preserving homeomorphism. It has an inverse $f_{k}^{-1}$, which is strictly increasing defined on $I_{k}$ such that $f_{k}^{-1}\left(x_{k}\right)=x_{k-1}$ and $f_{k}^{-1}\left(x_{k+1}\right)=x_{k}$. Thus, by (14) $f_{k+1}(x)$ is well defined on $\mathrm{I}_{k+1}$. Furthermore, by (15) we have

$$
\begin{aligned}
f_{k+1}\left(x_{k}\right) & =\lambda f_{k}\left(f_{k}^{-1}\left(x_{k}\right)+a\right)+\mu f_{k}^{-1}\left(x_{k}\right)+\varepsilon(a) \\
& =\lambda\left(x_{k}+a\right)+\mu x_{k-1}+\varepsilon(a)=x_{k+1} .
\end{aligned}
$$

Similarly, we also get $f_{k+1}\left(x_{k+1}\right)=x_{k+2}$. Thus,

$$
\begin{aligned}
f_{k+1}\left(x_{k}+a\right) & =\lambda f_{k}\left(f_{k}^{-1}\left(x_{k}+a\right)+a\right)+\mu f_{k}^{-1}\left(x_{k}+a\right)+\varepsilon(a) \\
& =\lambda f_{k}\left(x_{k-1}+2 a\right)+\mu\left(x_{k-1}+a\right)+\varepsilon(a) \\
& =\lambda x_{k+1}+\mu\left(x_{k-1}+a\right)+(4-3 \lambda) a \\
& =x_{k+1}+a
\end{aligned}
$$


and (15) is proved by induction.

Finally, for arbitrary $x \in\left(-\infty, x_{0}\right]$ by (13) there exists $n \in \mathbb{N}$ such that $x \in I_{n}$, where $\mathbb{N}$ denotes the set of all positive integers. Define

$$
f(x):=f_{n}(x) .
$$

Therefore, (14) and (16) lead to the fact

$$
f^{2}(x)=f_{n+1} \circ f_{n}(x)=\lambda f_{n}(x+a)+\mu x=\lambda f(x+a)+\mu x,
$$

which implies that the function defined by (16) is a continuous solution of Eq. $(10)$ on $\left(-\infty, x_{0}\right]$. This completes the proof.

Theorems 3-4 present a manner of construction for the continuous and unbounded solutions of Eq. (10) with $\varepsilon(a)=(4-3 \lambda) a$. Clearly, this construction is not unique, which depends on the chosen of $\varepsilon(a)$.

Example 4 Consider the equation

$$
f^{2}(x)=\frac{2}{3} f(x+a)+\frac{1}{3} x+2 a,
$$

where $\lambda=\frac{2}{3}, \mu=\frac{1}{3}$ and $\varepsilon(a)=2 a$.

All conditions in Theorems 3-4 can be verified respectively and therefore the equation has unbounded solutions on $(-\infty,+\infty)$.

\section{Acknowledgements}

The authors are grateful to the referee for his/her careful reading and comments. Project supported by the National Natural Science Foundation of China (11301226), Shandong Provincial Natural Science Foundation of China (ZR2014AL003), Scientific Research Fund of Shandong Provincial Education Department (J12L59) and Doctoral Fund of Binzhou University (2013Y04).

\section{References}

[1] M. Balcerowski, On the functional equation $x+f(y+f(x))=y+f(x+f(y))$, Aequationes Math., 75 (2008), 297-303.

[2] N. Brillouët-Belluot, Problem 15, Proceedings of 38th ISFE (2000 Hungary), Aequationes Math., 61 (2001), 304. 
[3] N. Brillouët-Belluot and Weinian Zhang, On a class of iterative-difference equations, J. Difference Equations Appl., 16 (2010), 1237-1255.

[4] Jingmin Chen and Weinian Zhang, Leading coefficient problem for polynomial-like iterative equations, J. Math. Anal. Appl., 349 (2009), 413-419.

[5] J. Dhombres, Some aspects of functional equations, Chulalongkorn University Press, Bangkok, 1979.

[6] W. Jarczyk, On an equation of linear iteration, Aequationes Math., 51 (1996), 303-310.

[7] J. Jarczyk, W. Jarczyk, On the problem of N. Brillouët-Belluot, Aequationes Math., 72 (2006), 198-200.

[8] M. Kuczma, On the mutual noncompatibility of homogeneous analytic non-power means, Aequationes Math., 45 (1993), 300-321.

[9] J. Matkowski, Weinian Zhang, On linear dependence of iterates, J. Appl. Anal., 6 (2000), 149-157.

[10] S. Nabeya, On the functional equation $f(p+q x+r f(x))=a+b x+c f(x)$, Aequationes Math., 11 (1974), 199-211.

[11] M. Sablik, Remark 4, Report of meeting, 10th International Conference on Functional Equations and Inequalities (Bedlewo, 2005), Ann. Acad. Paed. Cracoviensis Studia Math., 5 (2006), 127-165.

[12] J. Sikorska, Differentiable solutions of a functional equation related to the non-power means, Aequationes Math., 55 (1998), 146-152.

[13] J. Tabor, J. Tabor, On a linear iterative equation, Results Math., 27 (1995), 412-421.

[14] J. Tabor, M. Zoldak, Iterative equations in Banach spaces, J. Math. Anal. Appl., 299 (2004), 651-662.

[15] Bing Xu, Weinian Zhang, Construction of continuous solutions and stability for the polynomial-like iterative equation, J. Math. Anal. Appl., 325 (2007), 1160-1170.

[16] Dilian Yang, Weinian Zhang, Characteristic solutions of polynomial-like iterative equations, Aequationes Math., 67 (2004), 80-105. 
[17] Yingying Zeng, Weinian Zhang, Continuous solutions of an iterativedifference equation and Brillouët-Belluot's problem, Publ. Math. Debrecen, 78 (2011), 613-624.

[18] Weinian Zhang, On existence for polynomial-like iterative equations, Results Math., 45 (2004), 185-194.

[19] Weinian Zhang, K. Nikodem, Bing Xu, Convex solutions of polynomiallike iterative equations, J. Math. Anal. Appl., 315 (2006), 29-40.

Received: April 26, 2017 